
Verlag von VEIT \& COMP. in LEIPZIG, Marienstraße I8

\section{Funktionentheoretische Vorlesungen}

von Dr. Heinrich Burkhardt,

o. ö. Professor an der Techn. Hochechule Múnchen

$\mathrm{Z}$ wei Bände. Geh. 22 M. 60 Pf., geb. 28 M. 60 Pf.

\section{Erster Band}

\section{Heft. Algebraische Analysis}

Zweite, durchgeseh. u. vermehrte Anfl. Mit Fig. im Text. XI, 196 S. Geh. 5 M. 60 Pf., geb. 7 M. 60 Pf.

Il. Heft. Einfuhrung in die Theorie der analytischen Funktionen einer komplexen Verănderlichen

Vierte, durchgesehene Auflage. Mit zahlr. Fig. im Text. XII, 228 S. Geh. 7 M., geb. 9 M.

\section{$\mathrm{Z}$ weiter Band}

\section{Elliptische Funktionen}

Zweite, durchgesehene u. verb. Aufl. Mitzahlr. Fig. im Text. XVI, 384 S. Geh. 10 M., geb. 12 M.

„Als Antwort auf die Frage nach den Werken, die ein Kandidat vor der Prüfung studiert hat, erhält de: Professor regelmäBig die Titel der Burkhardtschen Schriften genannt. Und sie verdienen solche Verbreitung, weil sie einfach und klar abgefaBt sind und dennoch auf einem mäBigen Raume eine Fülle tiefen lnhalts bringen. Durch sie wird der Anfünger befähigt, in die neuen Erscheinungen der mathematischen Literatur mit Verständnis einzudringen “

Jabrbuch nber die Fortschritte der Mathruintik.

\section{Forschung und Studium}

Eine Sammlung mathematischer Monographien für Studierende von Dr. Gerhard Kowalewski,

Prof. an der Deutschen Universitat zu Prag.

Heft 1: Das Integral und seine geometrischen Anwendungen Mit Fig. IV, 86 S. Geh. 3 M.

„Das erste Heft bringt die moderne Behandlung der einfachen und der Doppelintegrale, und es wird darin z. B. die Transformation der Doppelintegrale in völlig strenger und doch ziemlich einfacher Wcisc erledigt.“ .Jahrbuch uber dle Fortschritte der Mathenatik.

\section{Vorlesungen \\ uber die Elemente der graphischen Statik von Dr. Friedrich Schur, Prot. an der Univ Straßburg,} herausgegeben unter Mitwirkung von W. Vogt VIII, $219 \mathrm{~S}$. Geh. $7 \mathrm{M}$., geb. $9 \mathrm{M}$.

Das Buch ist aus langjähriger Vorlesungspraxis an der Technischen Hochschule zu Aachen und Karlsruhe hervorgegangen. Will es daher einerseits den jungen Techniker mit der graphischen Behandlung der ebenen Statik bekannt und durch praktische Beispiele vertraut machen, so soll es andererseits den Mathematiker in die graphische Statik als geomctrische Disziplin einführen. 


\title{
VORLESUNGEN
}

\author{
ÜBER
}

\section{THERMODY NA MIK}

VON

\author{
DR. MAX PLANCK \\ PROFESSOR DER THEORETISCHEN PHYSIK \\ AN DER UNIVERSITÄT BERLIN.
}

FÜNFTE AUFLAGE.

MIT FÜNF FIGUREN IM TEXT.

\section{LEIPZIG}

VERLAG VON VEIT \& COMP. 
Druck von Metzger \& Wittig in Lelpzig. 